\title{
The contribution of visual and inertial mechanisms to navigation in total darkness
}

\author{
ETIENNE SAVE \\ Institute of Physiology, Czech Academy of Sciences, Prague, Czech Republic \\ and Centre de Recherche en Neurosciences Cognitives, CNRS, Marseille, France
}

\begin{abstract}
Rats were trained in the Morris water maze to reach a platform in darkness after they had been provided with some information on their initial location. They were given trials with (1) the light on (light condition) and (2) light followed by darkness (dark condition). In this latter condition, the dark period was preceded by either a brief (short group) or a long (long group) initial light period. Although the rats in the short group first oriented accurately toward the platform, their trajectory rapidly deviated in darkness. In contrast, the rats in the long group were able to navigate accurately over a longer distance, but finally also deviated. In transfer trials, the rats in the short group were less accurate than the rats in the long group in the dark; however, both groups performed well in light. This suggests that navigation in darkness depends not only on the use of recent visual and locomotor memory but also on the activation of an inertia-based navigation process.
\end{abstract}

Navigation is a behavior that uses most of an animal's sensory capabilities and is presumably controlled by external (visual, auditory, and olfactory) and internal (vestibular, proprioceptive, and somatosensory) cues. Earlier reports dealing with the sensory bases of spatial behaviors in rodents have shown that animals can use visual cues to define the location of a goal (Suzuki, Augerinos, \& Black, 1980; Zoladek \& Roberts, 1978) and to navigate toward a target (Collett, 1987; Collett, Cartwright, \& Smith, 1986; Morris, 1981). One way to study the importance of visual cues during navigation has been to assess the effects of limiting the availability of such cues on place learning. Sutherland, Chew, Baker, and Linggard (1987) have shown that the performance of rats in the Morris water maze task was impaired when the animals could not access distal visual information from the half of the pool containing the platform during learning. Moreover, these authors also found that illumination of the room during swimming, but not at the start or goal locations, was necessary for place learning. The effect of restricted visibility of distal visual cues on navigation is controversial. Devan, Blank, and Petri (1992) also found no effect of suppressing the visual cues when the animal reached and stayed on the platform. These results, however, are contrary to those of Whishaw (1991) and Arolfo, Nerad, Schenk, and Bures (1994), indicating that access

This work was supported by a fellowship from the Programme Cognisciences du Centre National de la Recherche Scientifique (France), by BMFT Grant O1 VG $9200215 / 26$, and by Grant 711401 from IGA of the Czech Academy of Sciences. The author would like to thank J. Bures, B. Poucet, C. Thinus-Blanc, and two anonymous reviewers for their comments, criticism, and suggestions. The author also thanks Y. Kaminsky for computer programming. Correspondence should be addressed to E. Save, Centre de Recherche en Neurosciences Cognitives, C.N.R.S., 31 Chemin J.-Aiguier, 13402 Marseille cedex 20, France (e-mail: espace@lnf.cnrs-mrs.fr). to visual information from the platform is necessary and sufficient to allow place learning.

Access to visual information during the initial stage of navigation, following release of the rat into the pool, may be crucial. Indeed, as soon as the animal is released in the water, it orients in a certain direction and then starts to swim. One hypothesis is that, during this very brief moment, the animal would be able to determine its position relative to the distal visual landmarks surrounding the pool and to plan its trajectory toward the goal. According to this hypothesis, rats would display accurate navigation in total darkness after they had the opportunity to determine their initial position in the environment. Such an ability has been demonstrated in the gerbil by Collett et al. (1986). One purpose of the present experiment was to assess whether and to what extent rats are able to plan and maintain accurate navigation in total darkness after they have been provided with environmental information about their starting location.

Because animals may rely mainly on distal visual cues to orient and initiate navigation, it might be assumed that they would only use their visual memory to maintain accurate navigation toward the goal in darkness. However, it has been shown that rodents are able to use a different navigational system to reach a goal in the absence of external cues (Etienne, Maurer, \& Séguinot, 1996; Gallistel, 1990). This system, called path integration or dead reckoning, is based on the processing of internal cues, such as vestibular, somatosensory, and proprioceptive cues. One property of this system is that it leads to an accumulation of error that limits the accuracy of navigation beyond short distances. Thus, if rats rely on the deadreckoning system in darkness, they should display a progressive decrease in accuracy over long distances.

In the present study, two groups of rats were trained on a place navigation task in the Morris water maze. Rats 
from each group were given trials in the standard condition of the Morris navigation task (i.e., with the room lights on; Morris, 1981). This was the light condition. It has been shown that learning to navigate in darkness following a light period is more efficient when the rats have been previously trained in total light (Arolfo et al., 1994). In alternation with the light condition, the rats were given trials in which an initial light period was followed by the onset of darkness (dark condition). During these trials, for one group, the room was illuminated during a short time corresponding to the very first stage of navigation that immediately follows release of the rats into the pool and during which they initiate swimming (Sutherland et al., 1987). For the second group, the room was illuminated for a longer time, allowing the rats to swim a certain distance with the light on. Once the training was complete, all rats were given test trials. They were released from novel starting points in order to test whether they would display accurate novel trajectories in light and in darkness. Because the capacity for accurate transfer has been debated with respect to cognitive mapping (O'Keefe \& Nadel, 1978) and associative learning (Sutherland \& Rudy, 1989) theories, such a test could give some hint about the mechanisms involved.

\section{METHOD}

\section{Subjects}

The subjects were 20 male Long-Evans hooded rats obtained from the breeding colony of the Institute of Physiology of the Czech Academy of Sciences. The animals weighed between 300 and $400 \mathrm{~g}$ at the start of the study. They were housed 3 per cage, with free access to food and water throughout the experiment.

\section{Apparatus}

Spatial learning. The water maze consisted of a large circular pool ( $2 \mathrm{~m}$ in diameter with $55-\mathrm{cm}$-high walls) filled to a depth of $25 \mathrm{~cm}$ with water maintained at $20^{\circ} \mathrm{C}$. The rats could escape from the water by climbing onto a cylindrical Plexiglas platform $(11 \mathrm{~cm}$ in diameter) that was constantly located in the middle of the northwest (NW) quadrant with its surface $1 \mathrm{~cm}$ below the water level. The pool was located in a large room containing numerous distal visual cues, including a door, a desk, a black curtain, and a shelf. Windows were covered in order to eliminate all sources of light during the dark period of the dark condition. In the light condition, the pool was indirectly illuminated by several lamps. All animals were tested between 0800 and $1200 \mathrm{~h}$.

During each daily session, the rats were given half of their trials in the light condition and half of their trials in the dark condition. For both conditions, a trial consisted of releasing a rat from a given starting point located at the periphery of the maze and continued until it reached the submerged platform. In the light condition, the animals were allowed to perform the task with the light always on. In the dark condition, two areas were defined in the pool: a central circular "dark area" surrounded by a ring-shaped "light area" (Figure 1). For one group, the light area corresponded to a narrow 15 . $\mathrm{cm}$-wide ring ( hhort group, $n=10$ ). For the other group, the light area corresponded to a larger $30-\mathrm{cm}$-wide ring (long group, $n=10$ ). The animals were released from the starting points while the room light was on (light area). The light was automatically turned off by the computer when the rat entered the dark area, and the room remained in complete darkness as long as the animal swam in this area (Figure 1). When the animal reached the target, the light was turned on. When the animal failed to reach the target,
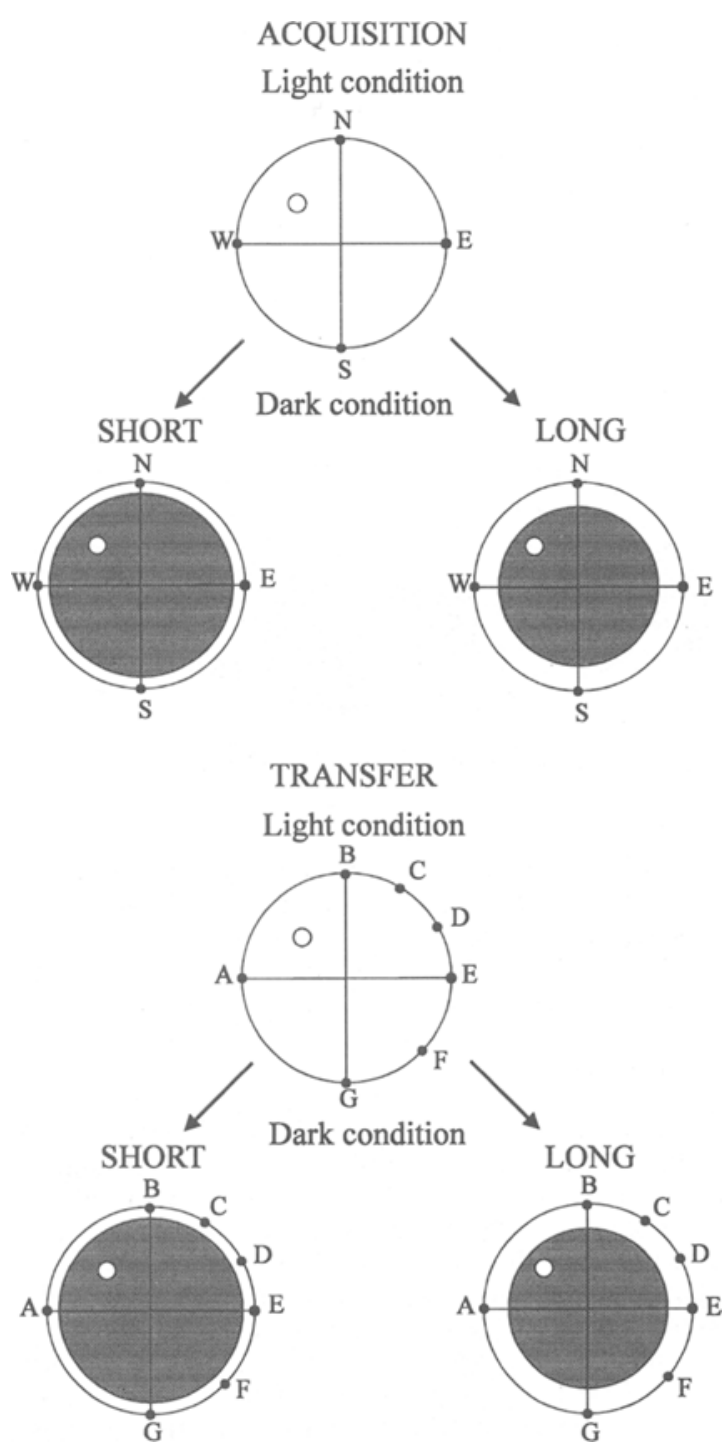

Figure 1. Experimental design. Each diagram represents a view of the water maze from above. During acquisition, the rats were trained to reach the platform located in the $N W$ quadrant from three starting places $(N, S$, and $W$ ) with the lights on (light condition) and in a condition involving an initial period of light followed by darkness (dark condition). In this latter condition, the rats in the long group were allowed to swim some distance from the starting point before the onset of darkness, whereas the rats in the short group were allowed to get only a visual snapshot of the environment before the onset of darkness. During transfer trials, the rats in each group were released from familiar $(A, B$, and $G$ ) as well as novel $(C, D, E$, and $F)$ starting points in both the light condition and the dark condition.

it eventually came out of the dark area, and the light was turned on. A similar procedure has been used by Arolfo et al. (1994), which allowed the animal to update its position in the environment and to make several attempts to reach the platform.

Tracking system. During each trial, whatever the illumination condition, the tested rat wore a rubber band around the chest to which was attached a Ping-Pong ball containing an infrared lightemitting diode (LED). As shown in Figure 2, this LED and its supply wires were attached to a counterweight mounted over the cen- 


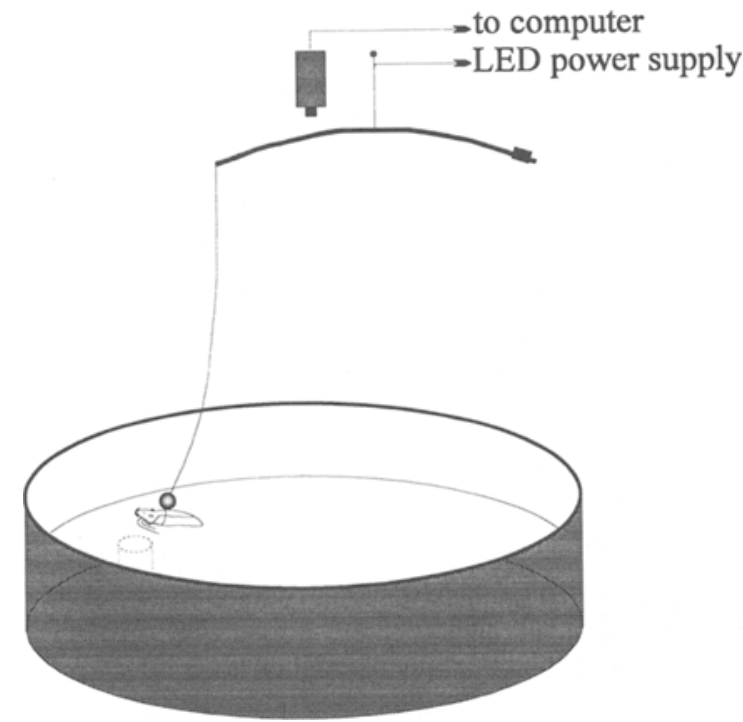

Figure 2. Apparatus and tracking system.

ter of the pool. The LED movements were monitored by an infrared TV camera positioned above the pool; every $100 \mathrm{msec}$, the computerized tracking system monitored and stored the $x-y$ coordinates of the LED. The TV monitor, power supply, and computer were located behind a black curtain, in an alcove, in one corner of the room.

\section{Behavioral Procedure}

Two groups of rats (short and long) were trained to reach the submerged platform, always located in the center of the NW quadrant, from three different, pseudorandomly determined, starting points (south [S], north [N], and west [W]). During each daily session, the animals were given 12 trials for 9 days, according to the following sequence: 3 trials with the light on (light condition), 3 trials with the light followed by darkness (dark condition), 3 trials in the light condition, and 3 trials in the dark condition. On a given trial, the rat was placed into the water, facing the wall, and was allowed to swim until it reached the hidden platform or until $60 \mathrm{sec}$ had elapsed. In the former case, when the rat climbed onto the platform, light was automatically turned on (in the dark condition), and the trial was over. In the latter case, the rat was manually led to the platform and was assigned the maximum latency of $60 \mathrm{sec}$. After each trial, the rat had a 5-sec rest on the platform before the next trial was run.

At Days 10 and 11 , the rats were given transfer trials. They were released from different starting points, including familiar points ( $A$, $B$, and $G$ ) and novel points (C, D, E, and F) (see Figure 1). From each starting point, the animals had to navigate a different distance before reaching the platform: $A$ and $B, 70 \mathrm{~cm} ; C, 90 \mathrm{~cm} ; D, 120 \mathrm{~cm}$; $E$ and $G, 130 \mathrm{~cm}$; and $F, 140 \mathrm{~cm}$. The rats were given 4 trials in the light condition, 3 trials in the dark condition, 3 trials in the light condition, and 4 trials in the dark condition from seven starting places pseudorandomly determined in each illumination condition.

\section{Data Collection and Analysis}

Average escape latency was calculated for each session, each group, and each illumination condition. Heading errors, defined as the angle between the target azimuth and the vector from the release point to the position of the animal, irrespective of the direction of deviation, were also calculated. In order to get a dynamic view of behavior and to describe the trajectories displayed by the animals in terms of accuracy with respect to the target, heading errors were calculated at different moments of navigation. In the light condition, heading errors were calculated for each successive sec- ond of swimming after start-up to 5 sec or until the animal reached the platform. In the dark condition, the first point of calculation corresponded to the border between the light area and the dark area. Successive heading errors were calculated at $1-\mathrm{sec}$ intervals up to $5 \mathrm{sec}$ or until the animal reached the platform. For calculation of group mean, the animals that had already reached the platform were given a heading error of zero.

Repeated measures analyses of variance (ANOVAs) were conducted on both the latencies and the heading errors using the VAR 3 program (Rouanet \& Lepine, 1970). This program also provided further comparisons and detailed analyses.

\section{RESULTS}

\section{Acquisition}

Escape latency. Mean escape latency across 9 days for the short and long groups in each illumination condition is shown in Figure 3. A $2 \times 2 \times 9$ (groups $\times$ illumination $X$ days) ANOVA, with repeated measures on the last factor, was conducted. Significant effects of group $[F(1,18)=136.83, p<.001]$, illumination $[F(1,18)=$ $148.38, p<.001]$, and day $[F(8,144)=88.78, p<.001]$ were obtained. There were significant effects of the interactions group $\times$ illumination $[F(1,18)=79.03, p<$ $.001]$, illumination $\times$ day $[F(8,144)=3.05, p<.01]$, group $\times$ day $[F(8,144)=3.18, p<.01]$, and group $\times$ illumination $\times$ day $[F(8,144)=2.27, p<.05]$. Detailed analyses showed that the rats in the short group displayed longer escape latency than did the rats in the long group in both the light condition and the dark condition $[F(1,18)$ $=12.90, p<.01$, and $F(1,18)=135.95, p<.001$, respectively]. This difference appeared in the early days of training [Day 3, $F(1,18)=15.12, p<.01]$ but disappeared by the end of training [e.g., Day $8, F(1,18)=$ 2.29 , n.s.]. In addition, the rats in both short and long groups showed longer escape latencies in the dark condition than in the light condition $[F(1,9)=138.02, p<$ .001 , and $F(1,9)=13.83, p<.01$, respectively]. However, for the rats in the long group, this difference was due to their extremely short latency and small standard error in the light condition rather than to high latency in

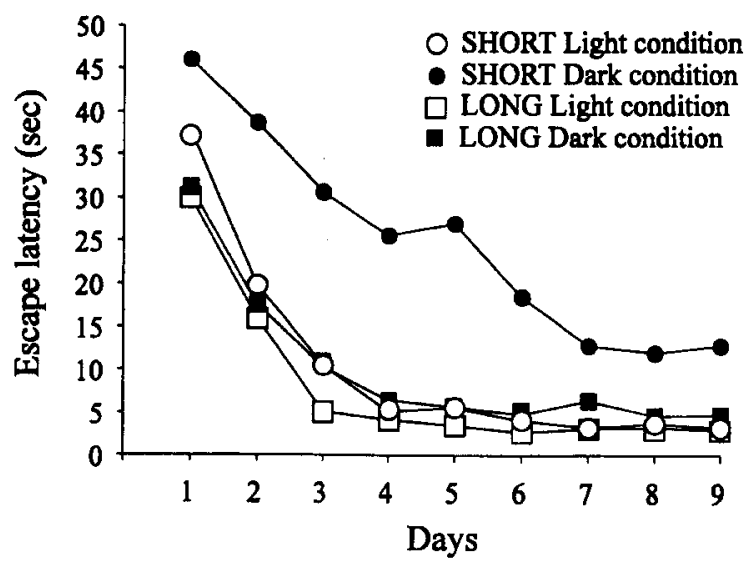

Figure 3. Mean escape latency to reach the platform during training. 
the dark condition. For instance, on Day 6 , in the light condition, the mean escape latency was $2.7 \pm 0.1 \mathrm{sec}$, and, in the dark condition, it was $4.9 \pm 0.6 \mathrm{sec}$. This indicates that the rats in the long group performed well in the dark.

In summary, (1) the short group in the dark condition displayed a slow rate of learning relative to in the light condition and relative to the long group in the dark condition, and (2) all groups improved their performance over days.

Heading errors. Four $2 \times 2 \times 3$ (groups $\times$ illumination $\times 3$-day blocks) ANOVAs, with repeated measures on the last factor, were conducted in order to compare heading errors in the short and long groups at four successive steps of navigation. The first step (hereafter called $\mathrm{Sec}$ 1) corresponded to the initial portion of swim in light (i.e., at $1 \mathrm{sec}$ after start for the light condition and at the border of the dark area for the dark condition) and the successive steps corresponded to $1-\mathrm{sec}$ intervals up to $4 \mathrm{sec}$. The time course of the mean heading error over the three blocks of acquisition is shown in Figure 4.

At all steps, the ANOVA revealed significant effects of group [Sec 1, $F(1,18)=8.74, p<.01 ; \operatorname{Sec} 2, F(1,18)=$ $32.71, p<.001 ; \operatorname{Sec} 3, F(1,18)=31.54, p<.001 ; \operatorname{Sec} 4$, $F(1,18)=67.74, p<.001]$, of block $[\operatorname{Sec} 1, F(2,36)=$ $88.68, p<.001 ; \operatorname{Sec} 2, F(2,36)=71.94, p<.001 ; \operatorname{Sec} 3$, $F(2,36)=72.18, p<.001 ; \operatorname{Sec} 4, F(2,36)=78.98, p<$
$.001]$, and, except for the 1st second, of illumination $[\operatorname{Sec} 1, F(1,18)=1.27$, n.s.; $\operatorname{Sec} 2, F(1,18)=19.77, p<$ $.001 ; \operatorname{Sec} 3, F(1,18)=63.06, p<.001 ; \operatorname{Sec} 4, F(1,18)=$ $55.22, p<.001]$. Only the group $\times$ illumination interaction was significant $[$ Sec $1, F(1,18)=13.11, p<.01$; $\operatorname{Sec} 2, F(1,18)=12.68, p<.01 ; \operatorname{Sec} 3, F(1,18)=13.70$, $p<.01 ; \operatorname{Sec} 4, F(1,18)=12.26, p<.01]$.

Detailed analyses revealed that, at the 1 st second (see Figure $4 \mathrm{~A}$ ), the rats in the short group displayed greater heading error than did the rats in the long group in the dark condition $[F(1,18)=17.34, p<.001]$, but not in the light condition $[F(1,18)=0.85$, n.s. $]$. Such a difference was confirmed for all blocks. This indicates that the rats in the long group better adjusted their trajectory than did the rats in the short group before the onset of darkness. Similarly, for all of the subsequent seconds (see Figure 4B, 4C, and 4D), although the difference between groups in the light condition fluctuated somewhat across blocks, at the end of training, the rats in the short group were less accurate than were the rats in the long group in the dark condition but not in the light condition [Sec 2 , Block 3, light condition, $F(1,18)=1.28$, n.s., and dark condition, $F(1,18)=7.19, p<.025$; Sec 3, Block 3 , light condition, $F(1,18)=3.30$, n.s., and dark condition, $F(1,18)=16.21, p<.001$; Sec 4 , Block 3 , light condition, $F(1,18)=2.75, \mathrm{n} . \mathrm{s}$., and dark condition, $F(1,18)=$ $19.65, p<.001]$. These results suggest that, throughout
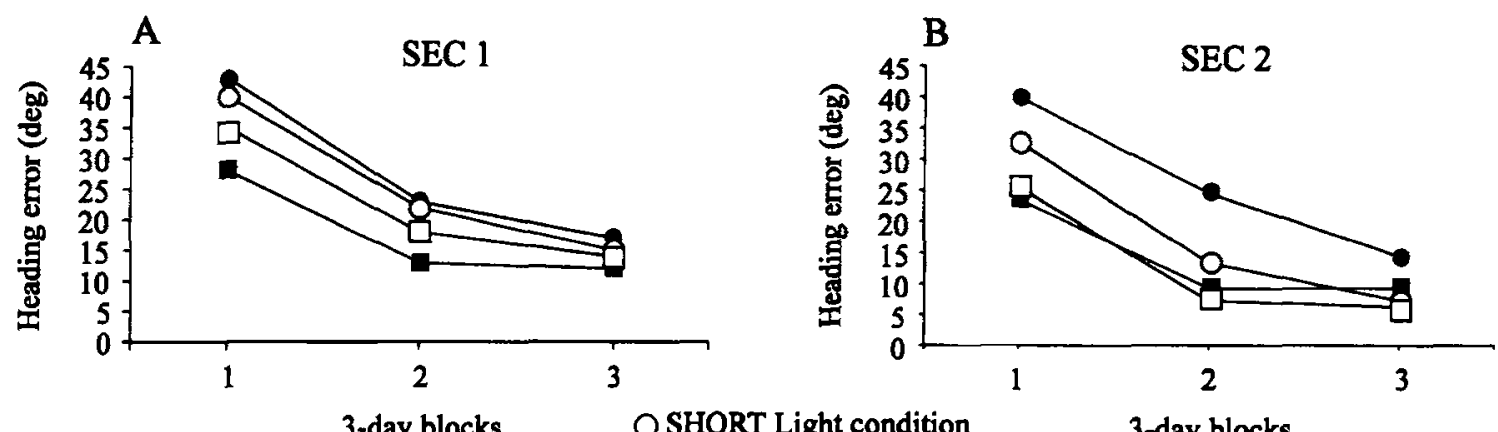

3-day blocks

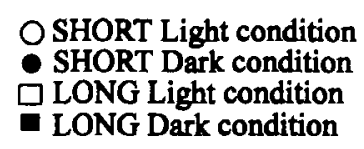

3-day blocks

D
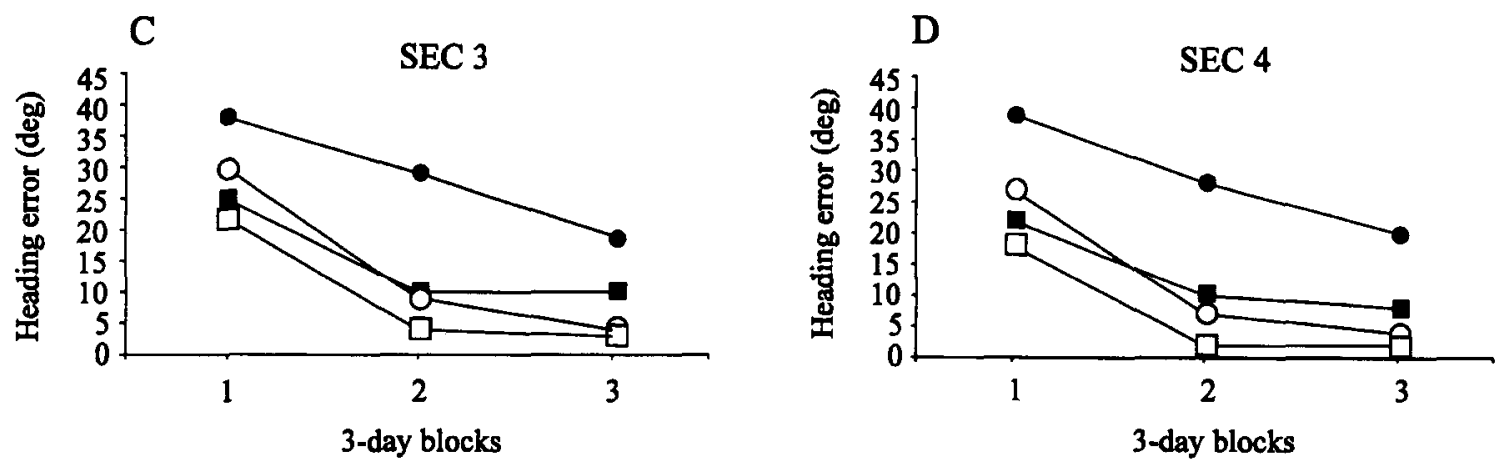

Figure 4. Heading errors for the three 3-day blocks of acquisition (Days 1-9). Heading errors have been calculated at (A) the 1 st second after start (light condition) and at the border of the dark zone (dark condition), (B) the 2nd second after start (light condition) and 1 sec after the onset of darkness (dark condition), (C) the 3rd second after start (light condition) and the 2 nd second after the onset of darkness (dark condition), and (D) the 4th second after start (light condition) and the 3rd second after onset of darkness (dark condition). 
navigation in darkness, the rats in the short group were less accurate than the rats in the long group.

Because it was assumed that performance in light reflects the greatest accuracy of the animals during navigation, we compared each group in both phases. In both groups, at Sec 1, all rats navigated under similar light illumination conditions. It was hypothesized that there would be no difference between the heading errors in the light and dark conditions. This hypothesis was confirmed for the rats in the short group $[F(1,9)=3.08$, n.s.], indicating that the access to environmental cues during the illuminated period allowed them to orient accurately toward the goal. Paradoxically, for the rats in the long group, heading error was higher in the light condition than in the dark condition $[F(1,9)=11.40, p<.01]$. However, at the end of training (Block 3 ), these rats were not different in both phases $[F(1,9)=0.56$, n.s.].

For the subsequent seconds, heading errors were compared as the animals swam in the dark (dark condition) or in the light (light condition). At Sec 2, the rats in the short group were found to be less accurate in the dark condition than in the light condition $[F(1,9)=21.66$, $p<.001]$. In contrast, the rats in the long group were still as accurate in the dark condition as in the light condition $[F(1,9)=0.75$, n.s. $]$. At Sec 3 and Sec 4 , the rats in both groups displayed higher heading errors in the dark condition than in the light condition [short group, $\operatorname{Sec} 3, F(1,9)=42.22, p<.001$, and $\operatorname{Sec} 4, F(1,9)=$ $34.90, p<.001$, long group, $\operatorname{Sec} 3, F(1,9)=22.76, p<$ .01 , and $\operatorname{Sec} 4, F(1,9)=26.86, p<.001]$.

It is interesting to compare the performance of the rats in the short and long groups after they learned the task (Block 3). The accuracy of each group at successive seconds of swimming is shown in Figure 5 . A $2 \times 2 \times 4$ (groups $\times$ illumination $\times$ seconds) ANOVA, with repeated measures on the last factor, revealed effects of group $[F(1,18)=14,25, p<.01]$, illumination $[F(1,18)$ $=115.09, p<.001]$, and second $[F(3,54)=30.24, p<$ $.001]$. Detailed comparisons showed that there was no difference between the groups at Sec 1. Nevertheless, the two groups were found to deviate from their corresponding trajectory in light from Sec 2 up to Sec 4 [e.g., on Sec 2, short group, $F(1,9)=45.00, p<.001$, and long group, $F(1,9)=7.35, p<.05]$. However, most importantly, the rats in the short group were clearly less accurate than the rats in the long group in the dark condition $[F(1,18)=22.18, p<.001]$, but not in the light condition $[F(1,18)=1.74$, n.s. $]$.

In summary, (1) the rats in the short group were more impaired than the rats in the long group in adjusting their trajectory in the dark condition (they also displayed an important deviation with respect to their trajectory in light), (2) the rats in the long group deviated somewhat from their corresponding trajectory in light, but their performance remained quite good throughout navigation, and (3) all groups improved over days.

Although the view of the environment was very brief for the rats in the short group, they initially oriented correctly toward the platform. However, this accuracy was

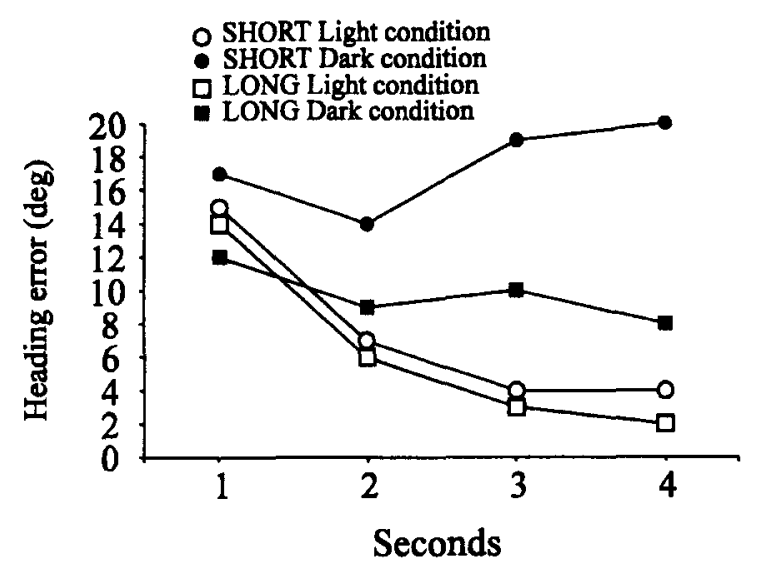

Figure 5. Heading errors at $\operatorname{Sec} 1, \operatorname{Sec} 2, \operatorname{Sec} 3$, and $\operatorname{Sec} 4$ for the third 3-day block of training during acquisition.

found to deteriorate rapidly with the distance swum in darkness. The rats in the long group were able to maintain good accuracy, although they deviated in the dark from their trajectory in light. Figures 6 and 7 display the initial parts of trajectories for representative animals at Day 1 and Day 9 of training. This shows that, at the end of training (Day 9), the rats in both groups performed as well in the dark as in the light for short distances. Because, in the acquisition phase, accuracy was averaged across three starting points including one long and two short paths, the second part of the study was aimed at (1) assessing navigation in darkness over different distances and (2) testing whether the animals could transfer their learning to novel starting places. The animals were given transfer trials from several starting points including familiar and novel ones.

\section{Transfer Trials}

Escape latency. Mean escape latency for each starting point for the short and long groups in both the light condition and the dark condition is displayed in Figure 8. A $2 \times 2 \times 5$ (groups $\times$ illumination $\times$ starting points) ANOVA revealed effects of group $[F(1,18)=15.67, p<$ $.001]$, illumination $[F(1,18)=54.90, p<.001]$, and starting point $[F(4,72)=13.58, p<.001]$. Only the group $\times$ illumination interaction was significant $[F(1,18)$ $=14.03, p<.01]$. Detailed comparisons showed that the rats in the short group displayed higher escape latency than did the rats in the long group only in the dark condition $[F(1,18)=15.73, p<.001]$. Moreover, for both groups, escape latencies were higher in the dark condition than in the light condition [short group, $F(1,9)=$ 43.94, $p<.001$; long group, $F(1,9)=11.49, p<.001]$.

In summary, as in the acquisition, (1) the rats in the short group displayed a longer escape latency than did the rats in the long group in the dark condition and (2) the rats in the long group displayed higher escape latency in the dark condition than in the light condition.

Heading errors. Figure 9 shows, for each starting point, the time course of heading errors over $5 \mathrm{sec}$ of swimming for both groups in the light and dark condi- 


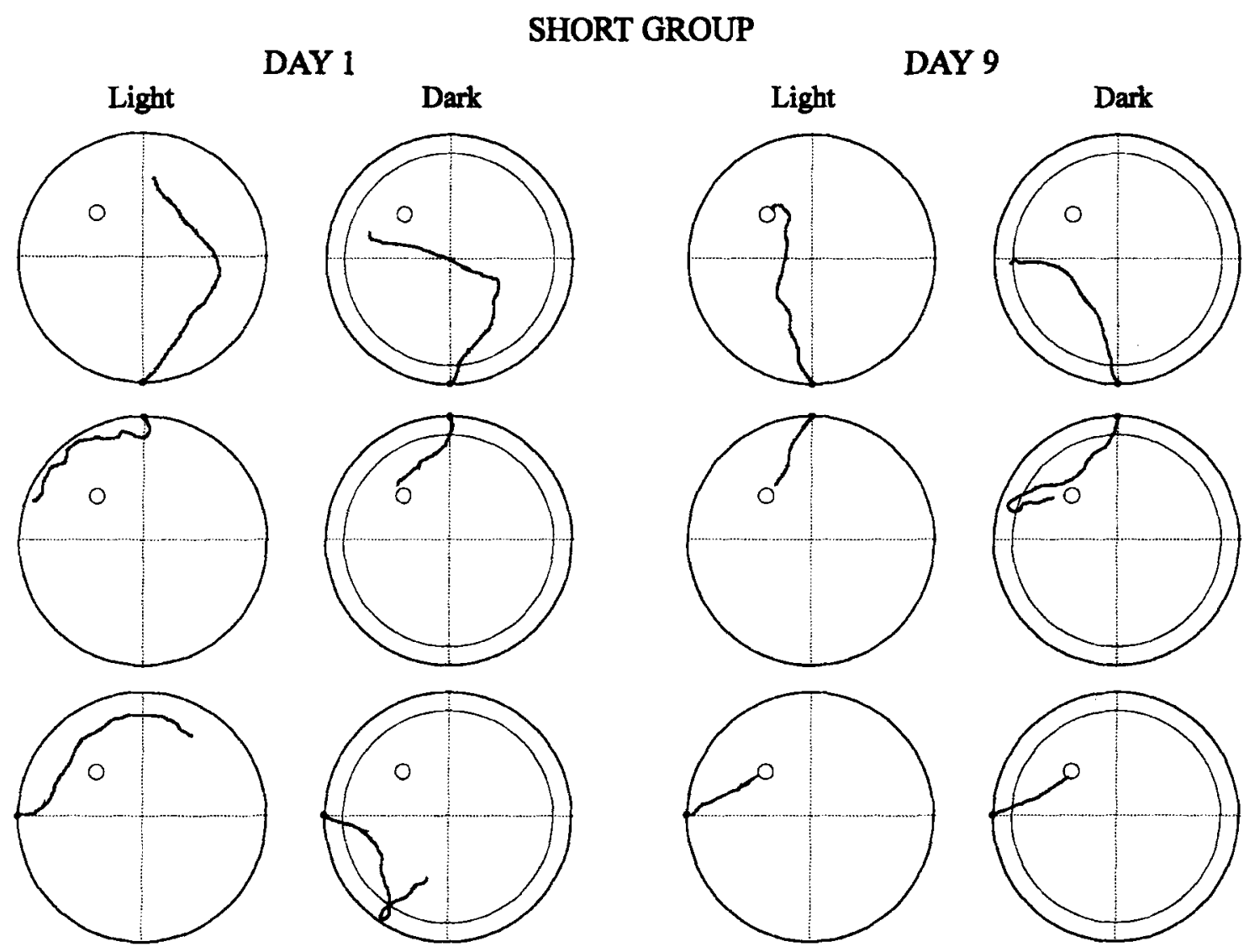

Figure 6. Representative swimming trajectories for the short group. For each diagram, the outer circle represents the edge of the water maze and the inner circle represents the limit between the light area and the dark area. The black dot on the outer circle shows the starting point. Each diagram displays the initial part of the trajectories: the five 1st seconds of swimming or less when the rat reached the platform before the $5 \mathrm{sec}$ had elapsed. To show acquisition of these trajectories, Day 1 (beginning of training) and Day 9 (end of training) are displayed.

tions. For all starting points, the rats in the two groups initially oriented toward the platform as well as, if not better than, at the end of the training session. Data were therefore pooled according to the distance from the platform (see Figure 1). In order to evaluate accuracy of navigation from each starting location (A/B, C, D, E/G, and F), five $2 \times 2 \times 5$ (groups $\times$ illumination $\times$ seconds) ANOVAs, with repeated measures on the last factor, were conducted on the data.

For most of the starting places (A/B, C, D, and E/G), the short and long groups were not different $[F(1,18)<$ $4.41, p>.05]$. Only for starting point $F$ was there an effect of group $[F(1,18)=8.48, p<.01]$, due to the fact that the rats in the short group displayed much higher heading errors than did the rats in the long group in the dark condition $[F(1,18)=13.05, p<.01]$. The illumination factor had no consistent effect on the performance (it was significant for starting points $\mathrm{C}$ and $\mathrm{E} / \mathrm{G}$, but not for the others). However, there was an effect of the group $X$ illumination interaction for the two starting points associated with the longest paths $[\mathrm{E} / \mathrm{G}, F(1,18)=5.60$, $p<.05 ; \mathrm{F}, F(1,18)=4.69, p<.05]$. There was an effect of second for all the starting points $[\mathrm{A} / \mathrm{B}, F(4,72)=9.84$, $p<.001 ; \mathrm{C}, F(4,72)=11.61, p<.001 ; \mathrm{D}, F(4,72)=$ $29.44, p<.001 ; \mathrm{E} / \mathrm{G}, F(4,72)=10.22, p<.001 ; \mathrm{F}$, $F(4,72)=5.18, p<.01]$. The illumination $\times$ second interaction was significant for all of the starting points except $\mathrm{C}[\mathrm{e} . \mathrm{g} ., \mathrm{A} / \mathrm{B}, F(4,72)=9.95, p<.001 ; \mathrm{E} / \mathrm{G}, F(4,72)=$ $45.24, p<.001]$.

Detailed comparisons confirmed that, for starting points $A / B$ and $C$, each group displayed a similar heading error in the light condition and in the dark condition throughout navigation [all $F_{\mathrm{s}}(1,18)<4.41, p \mathrm{~s}>.05$ ]. For starting points $D, E / G$, and $F$, no difference was found in the light condition, whereas the rats in the short group displayed higher heading error than did the rats in the long group in the dark condition $[\mathrm{D}, F(1,18)=4.64$, $p<.05 ; \mathrm{E} / \mathrm{G}, F(1,18)=6.48, p<.05 ; \mathrm{F}, F(1,18)=$ $13.04, p<.01]$. In light, all rats were able to correct their trajectories, whereas, in darkness, the rats in the short group deviated from their trajectory in light for starting places $\mathrm{D}, \mathrm{E} / \mathrm{G}$, and $\mathrm{F}[\mathrm{D}, F(1,9)=5.63, p<.05 ; \mathrm{E} / \mathrm{G}$, $F(1,9)=38.12, p<.001 ; \mathrm{F}, F(1,9)=6.83, p<.05]$. For all of these starting points, this deviation became significant at $\operatorname{Sec} 3, \operatorname{Sec} 4$, and $\operatorname{Sec} 5$. In darkness, the rats in the long group were found to deviate from their trajec- 


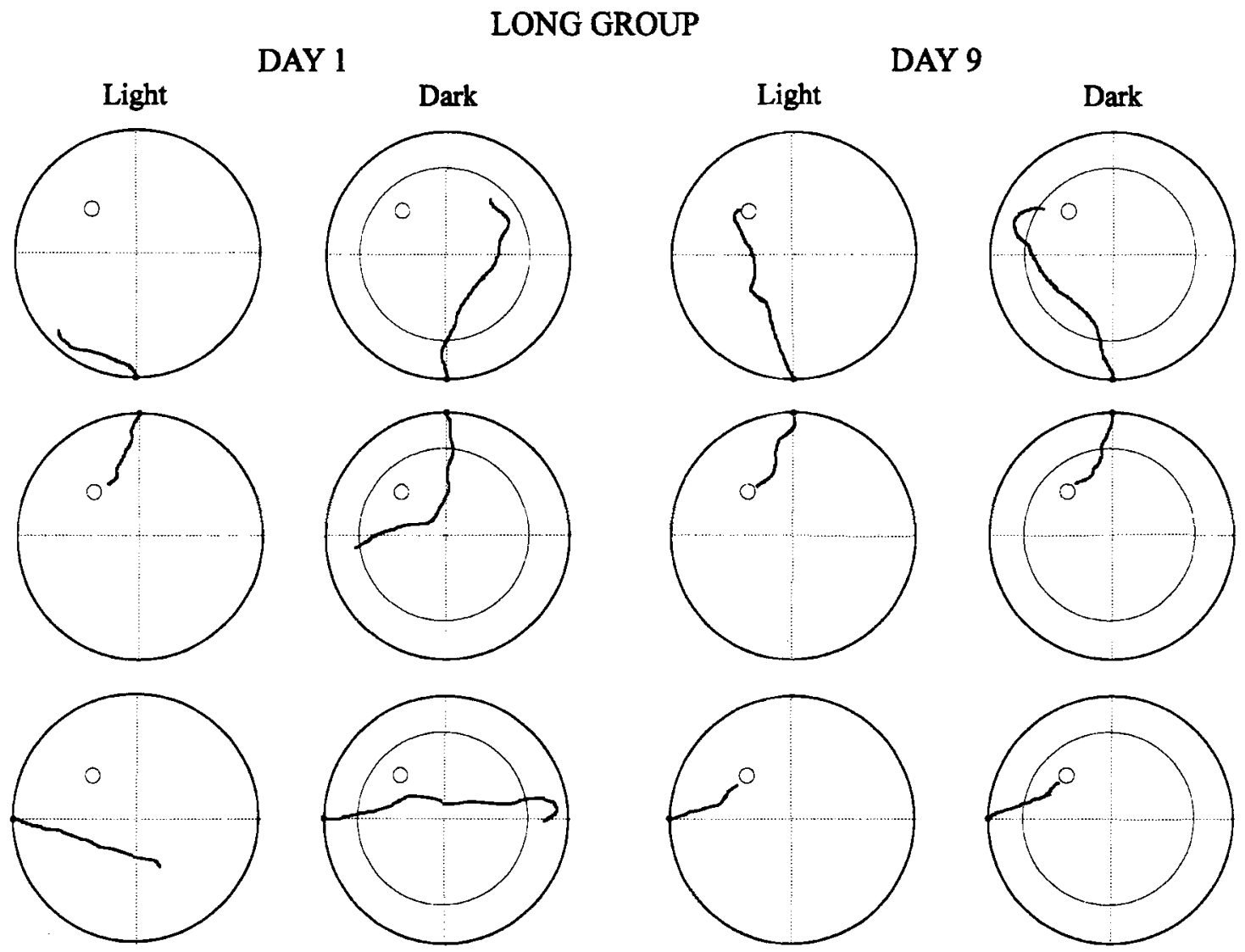

Figure 7. Representative swimming trajectories for the long group. Trajectories are shown as in Figure 6.

tory in light at $\operatorname{Sec} 3, \operatorname{Sec} 4$, and $\operatorname{Sec} 5$ for starting point $\mathrm{E} / \mathrm{G}$ and at Sec 5 for starting point $\mathrm{F}$. It is important to note that, in the light condition, all rats swam successfully to the platform, displaying low latency (Figure 8) and heading error (Figure 9). Figure 10 displays the initial parts of trajectories from each novel starting point for representative animals.

\section{DISCUSSION}

In this experiment, we attempted to assess the accuracy of navigation in total darkness after rats determined their initial position in light. The rats were trained in a place navigation task either in a light condition or in a dark condition (light followed by dark). During this latter condition, the room was illuminated only during the first stage of navigation that immediately followed release of the rat into the pool and during which it initiated swimming (short group) or it was illuminated for a longer time, allowing the animal to swim farther with the light on (long group).

The results showed that the rats in the short group were not able to maintain an accurate trajectory in darkness. They were found to deviate from their corresponding trajectory in light soon after the lights went off. In contrast, the rats in the long group were more accurate than the rats in the short group and displayed fairly good accuracy, although they were not as accurate in the darkness as in the light. This shows that there is a beneficial effect of swimming in light for accurate navigation in darkness. For the short group, a possible interpretation of this impairment is that the rats were unable to process spatial information and then plan their trajectory. In-

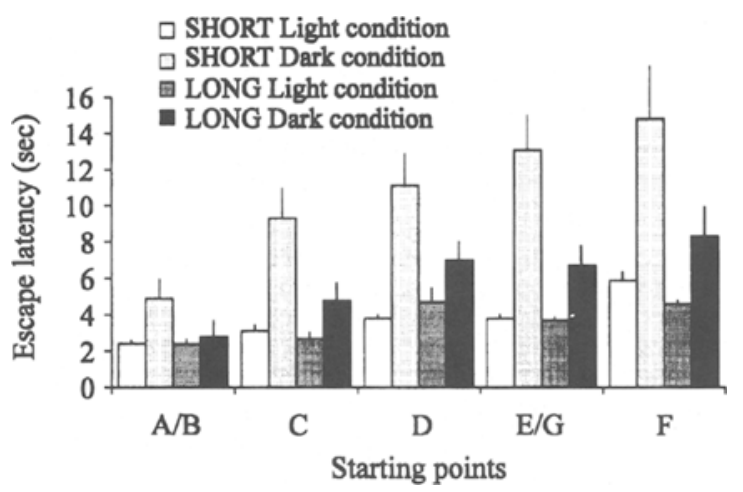

Figure 8. Mean escape latency $(+S E M)$ to reach the platform from different starting points during the transfer trials (see Figure 1). The data concerning the starting points located at the same distance from the platform have been pooled $(A / B$ and E/G). 

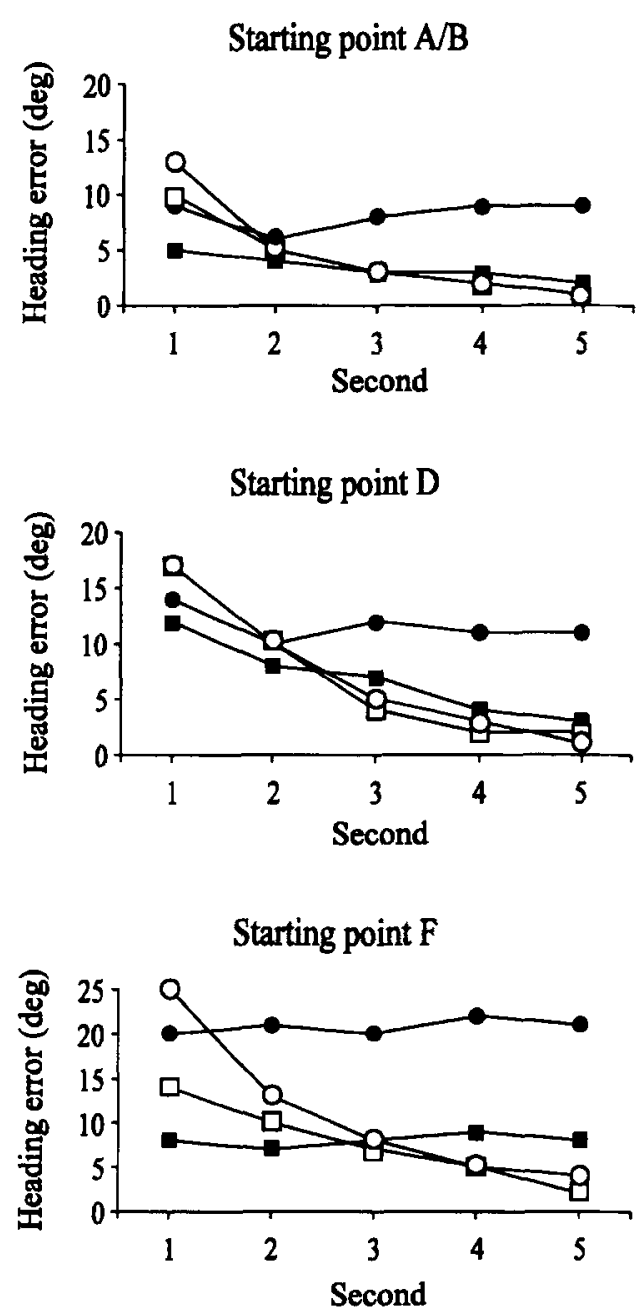
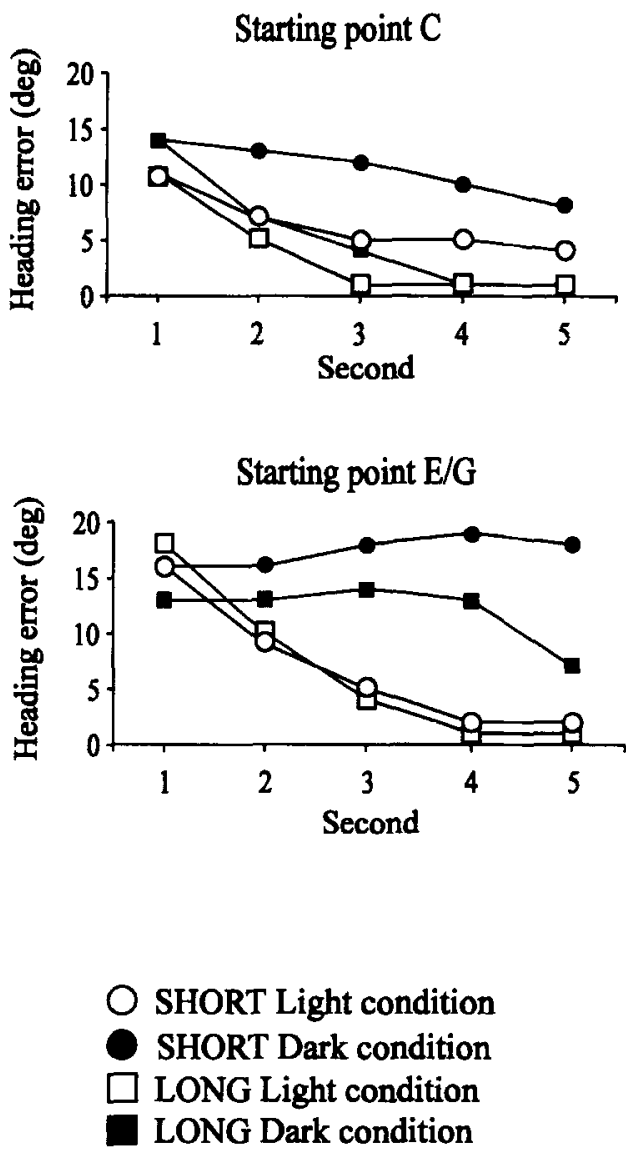

Figure 9. Heading errors during the transfer trials involving different starting points (see Figure 1). The data for starting points located at the same distance from the platform have been pooled ( $\mathrm{A} / \mathrm{B}$ and $\mathrm{E} / \mathrm{G})$. Heading errors were calculated for each of the 5 successive seconds after start (light condition) or after the onset of darkness (dark condition).

deed, at the start, the rats were placed into the pool facing the wall and then had to make a $180^{\circ}$ turn to orient toward the target. These movements could have prevented the animals from relying on distal landmarks. However, such a hypothesis, plausible for the first block of learning trials, has to be discarded when considering the third block. At the end of acquisition, the rats in the short group displayed good initial accuracy in both the light condition and the dark condition. Therefore, the results suggest that the rats in the short group (like those in the long group), although not able to maintain an accurate navigation in darkness, were able to plan their trajectory before the onset of darkness. Such place recognition and trajectory planning may be accounted for by the use of local views of the environment. A local view is defined as a two-dimensional panorama from a particular vantage point (Cartwright \& Collett, 1982; Poucet, 1993; Zipser, 1986). To recognize a place, the animal has to compare the current local view to previously stored local views (Collett et al., 1986). Such matching at the starting point would allow the animals to orient correctly and to eventually compute a trajectory. Trajectory planning was correct for both groups, but only the rats in the long group were able to maintain a correct trajectory after the onset of darkness.

Associative learning processes may also account for place navigation (Sutherland \& Rudy, 1989). It has been proposed that an association between successive local views and specific movements could allow place recognition and place navigation (Kuipers, 1982; McNaughton, Leonard, \& Chen, 1989). According to this hypothesis, rats should display accurate transfer only after they have been allowed to experience all the cues (visual and kinesthetic) useful to master the task (Whishaw, 1991). Relevant cues are those perceived from the goal location and those that are generated by the animal's movements as it swims to the goal (Sutherland et al., 1987). In our experiment, although the rats could swim freely all around the 
Starting points $\mathrm{C}$

D

$\mathbf{E}$

$\mathbf{F}$

\section{LONG}
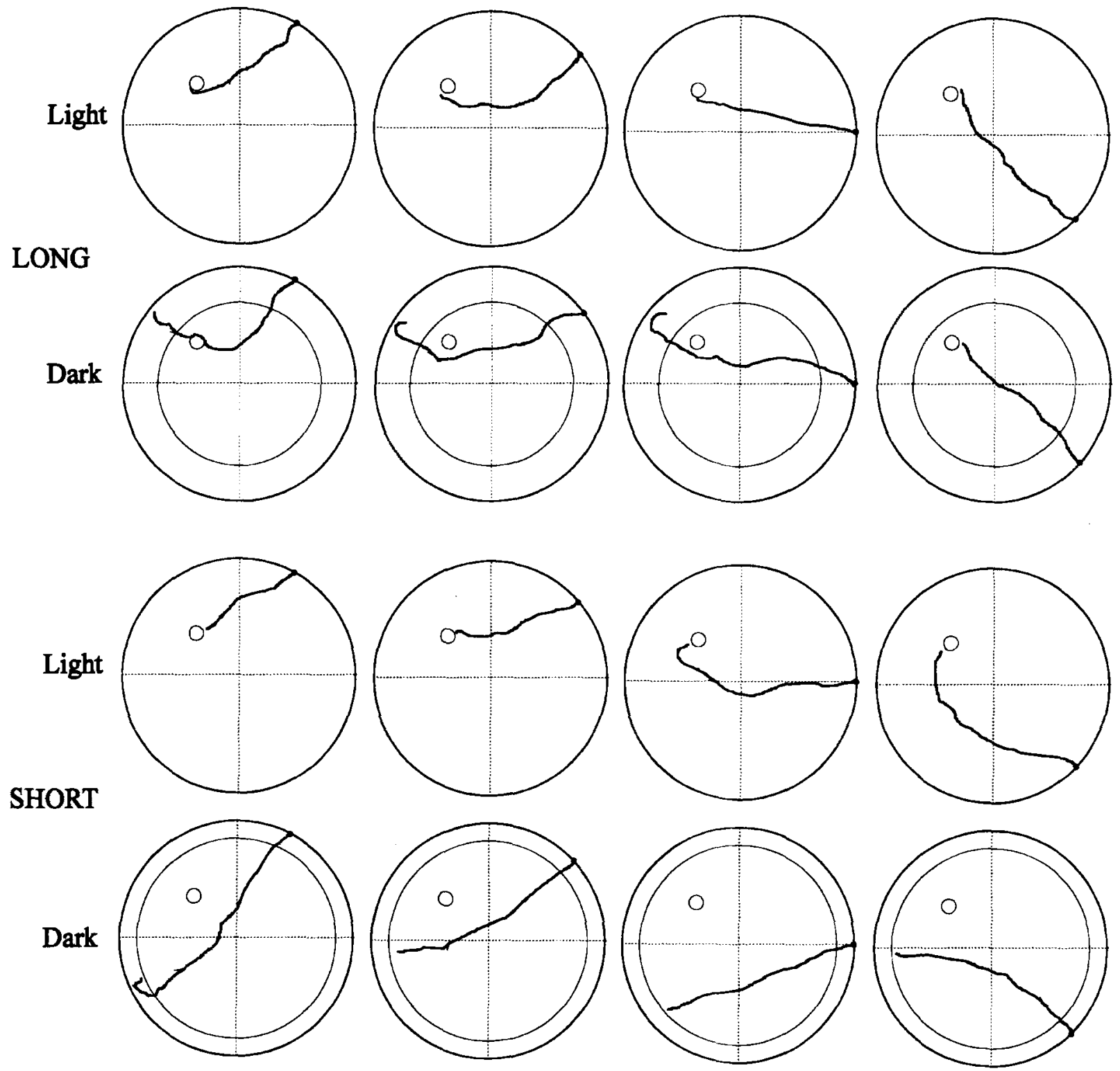

Figure 10. Representative swimming trajectories for the short and long groups during the transfer trials (the trajectories from the starting points $C, D, E$, and $F$ are displayed). Trajectories are shown as in Figure 6.

pool during early trials, it seems doubtful that they could associate specific views or guiding cues with specific trajectories leading to the goal. Our rats learned quickly to go directly to the platform from the training starting points, and they spent little time in the rest of the pool. Moreover, they displayed high transfer performance in light, since they reached the platform as early as the first transfer trial with low latency and heading error. In addition, the rats in both groups immediately displayed an accurate initial orientation during transfer trials in the dark condition. They also navigated accurately over short distances in the dark. Associative learning thus cannot explain completely the accuracy of navigation during the acquisition and transfer trials in the light and dark conditions.

We consider next the hypothesis that, during learning, the animals used environmental landmarks to form a representation of the environment (a cognitive map) that allowed them to anticipate landmark configurations seen from different vantage points and, thus, to locate the goal from places not yet visited (O'Keefe \& Nadel, 1978). The formation of such a representation does not exclude the possible use of local views (Poucet, 1993). Both theories predict that the rats would navigate accurately in darkness provided that they had some visual information 
about their starting place. In our experiment, this prediction was not confirmed, because the rats in the short group in darkness deviated considerably from their trajectory in light, and the rats in the long group-although able to maintain a fairly good trajectory - displayed a persistent error and missed the platform more frequently than in light. It could be argued that if we had let the rats swim a little longer in light, they would have reached the platform. Arolfo et al.'s results show that this is not the case, because, although their rats were allowed to swim $50 \mathrm{~cm}$ before the onset of darkness, they were impaired in place learning (Arolfo et al., 1994). Our results suggest that the map (which was used in the light condition and in the dark condition) is not precise enough to allow accurate performance in the dark, regardless of the initial amount of information processed in the light (see also Whishaw, 1991, for a similar idea).

In darkness, rats cannot use visual feedback to guide their course and adjust their trajectory, but they may rely instead on internal cues - such as vestibular, proprioceptive, and somatosensory cues-to navigate and reach the platform. A critical feature of navigation based on the processing of inertial information is that its accuracy is limited due to the problem of cumulative errors. Therefore, if rats rely on this process to navigate in the dark, they should deviate progressively from their trajectory. Such behavior was observed in the rats in the long group that deviated progressively from their corresponding trajectory in light. It has been widely demonstrated that, in absence of external cues, rodents are able to update their position with respect to their starting place by integrating the internal cues generated during locomotion (Etienne et al., 1996; Gallistel, 1990; Mittelstaedt \& Mittelstaedt, 1980). This dead-reckoning or path-integration process has been proposed to be part of a basic automatic navigational system. It can be hypothesized, therefore, that the inaccuracy of navigation in darkness could be due to the inability to maintain a trajectory on an inertial basis. Beyond a certain distance, maintaining an accurate trajectory would require viewing the environment (Gallistel, 1989; McNaughton, Chen, \& Markus, 1991), particularly in the vicinity of the platform (Arolfo et al., 1994).

Interestingly, recent studies, at the neural level, have emphasized that rats could keep track of their position in total darkness (McNaughton et al., 1989; Quirk, Muller, \& Kubie, 1990). In these studies, hippocampal "place cells" were recorded in the freely moving rat. These cells are active only when the animal is in a particular place in its surrounding (Muller \& Kubie, 1987), and the characteristic firing pattern of these cells is the "place field" (O'Keefe \& Dostrovsky, 1971). It has been demonstrated that place cell firing patterns persisted in total darkness only when the dark period was preceded by a light period. The stability of place fields in darkness seemed to be dependent on visual memory of the starting place and on the updating of the animal's position with kinesthetic - mainly vestibular-information.
Overall, the results of the present study support the idea that navigation depends on an interplay between the dead-reckoning system and visual information provided by stable landmarks (Etienne, Joris-Lambert, Reverdin, \& Teroni, 1993; Etienne et al., 1996; Gallistel, 1989). In light, rats benefit from initially viewing the environment, and they adjust their trajectory toward the target. In darkness, they probably rely on the memory of their initial trajectory to maintain accurate navigation. The progressive inaccuracy suggests, nevertheless, that rats also rely on kinesthetic cues and use the dead-reckoning system. The interplay between vision and the dead-reckoning system is further required to correct the heading error and maintain navigation over longer distances.

\section{REFERENCES}

Arolfo, M. P., Nerad, L., Schenk, F., \& Bures, J. (1994). Absence of snapshot memory of the target view interferes with place navigation learning by rats in the water maze. Behavioral Neuroscience, 108, 308-316.

Cartwright, B. A., \& Collett, T. S. (1982). How honey bees use landmarks to gujde their return to a source food. Nature, 295, 560564.

COLlEtT, T. S. (1987). The use of visual landmarks by gerbils: Reaching a goal when landmarks are displaced. Journal of Comparative Physiology A, 160, 109-113.

Collett, T. S., Cartwright, B. A., \& Smith, B. A. (1986). Landmark learning and visuo-spatial memories in gerbils. Journal of Comparative Physiology (A), 158, 835-851.

Devan, B. D., Blank, G. S., \& Petri, H. L. (1992). Place navigation in the Morris water task: Effects of reduced platform interval lighting and pseudorandom platform positioning. Psychobiology, 20, 120126.

Etienne, A. S., Joris-Lambert, S., Reverdin, B., \& Teroni, E. (1993). Learning to recalibrate the role of dead reckoning and visual cues in spatial navigation. Animal Learning \& Behavior, 21, 266-280.

Etienne, A. S., Maurer, R., \& SÉGuinot, B. (1996). Path integration in mammals and its interaction with visual landmarks. Journal of Experimental Biology, 199, 201-209.

GALLISTEL, C. R. (1989). Animal cognition: The representation of space, time and number. Annual Reviews of Psychology, 40, 155-189.

GaLlistel, C. R. (1990). The organization of learning. Cambridge, MA: MIT Press.

KUIPERS, B. (1982). The "map in the head" metaphor. Environment \& Behavior, 14, 202-220.

McNaughton, B. L., Chen, L. L., \& Markus, E. J. (1991). "Dead reckoning," landmark learning, and the sense of direction: A neurophysiological and computational hypothesis. Journal of Cognitive Neuroscience, 3, 190-202.

McNaughton, B. L., Leonard, B., \& Chen, L. (1989). Corticalhippocampal interactions and cognitive mapping: A hypothesis based on reintegration of the parietal and inferotemporal pathways for visual processing. Psychobiology, 17, 236-246.

Mittelstaedt, M. L., \& MitTelstaedt, H. (1980). Homing by path integration in a mammal. Naturwissenschaften, 67, 566 .

MorRIS. R. G. M. (1981). Spatial localization does not require the presence of local cues. Learning \& Motivation, 12, 239-260.

Muller, R. U., \& Kubie, J. L. (1987). The effect of changes in the environment on the spatial firing of hippocampal complex-spike cells. Journal of Neuroscience. 7, 1935-1950.

O'KeEFE, J., \& Dostrovsky, J. (1971). The hippocampus as spatial map: Preliminary evidence from unit activity in the freely moving rat. Brain Research, 34, 171-175.

O'KeEFE, J., \& NADEL, L. (1978). The hippocampus as a cognitive map. Oxford: Oxford University Press. 
POUCET, B. (1993). Spatial cognitive maps in animals: New hypotheses on their structure and neural mechanisms. Psychological Review, 100, 163-182.

Quirk, G. J., Muller, R. U., \& Kubie, J. L. (1990). The firing of hippocampal place cells in the dark depends on the rat's recent experience. Journal of Neuroscience, 10, 2008-2017.

RoUANET, H., \& LEPINE, D. (1970). Comparisons between treatment in a repeated measurement design: ANOVA and multivariate methods. British Journal of Mathematical \& Statistical Psychology, 23, 146-163.

Sutherland, R. J., Chew, G. L., BaKer, J. C., \& LingGard, R. C. (1987). Some limitations on the use of distal cues in place navigation by rats. Psychobiology, 15, 48-57.

SutHERLAND, R. J., \& RUDY, J. W. (1989). Configural association theory: The role of the hippocampal formation in learning, memory, and amnesia. Psychobiology, 17, 129-144.

Suzuki, S., Augerinos, G., \& Black, A. H. (1980). Stimulus control of spatial behavior on the eight-arm maze in rats. Learning \& Motivation, 11, 1-18.

WhISHAw, I. Q. (1991). Latent learning in a swimming pool place task by rats: Evidence for the use of associative and not cognitive mapping processes. Quarterly Joumal of Experimental Psychology, 43B, 83-103.

ZIPSER, D. (1986). Biologically plausible models of place recognition and goal location. In J. L. McClelland, D. E. Rumelhart, \& the PDP Research Group (Eds.), Parallel distributed processing: Explorations in the microstructure of cognition. Vol 2: Psychological and biological models (pp. 433-469). Cambridge, MA: MIT Press.

ZOLADEK, L., \& RoBERTS, W. A. (1978). The sensory basis of spatial memory in the rat. Animal Learning \& Behavior, 6, 77-81.

(Manuscript received April 22, 1996; revision accepted for publication February 20, 1997.) 